\section{Lebenszyklusbasierte Nachhaltigkeitsbewertung von Produkten}

\author{
von Walter KIöpffer, LCA Consult \& Review, \\ Frankfurt a. M., und Isa Renner, Energie- \\ und Umweltberatung, Rüsselsheim
}

\begin{abstract}
Nachhaltigkeit wurde vom United Nations Environment Programme (UNEP) in Rio als politisches Hauptziel der zukünftigen Entwicklung der Menschheit postuliert. Daher sollte auch eine nachhaltige Produktentwicklung angestrebt werden. Nach der allgemein bekannten Interpretation der Originaldefinition aus dem Brundtland-Report beinhaltet Nachhaltigkeit drei Komponenten: Umwelt, Ökonomie und soziale Aspekte. Es liegt in der Verantwortlichkeit der beteiligten Wissenschaftler, ein angemessenes, zuverlässiges und aktuelles Instrument zur Nachhaltigkeitsbewertung bereit zu halten. Dabei ist unbedingt darauf zu achten, dass sich die Instrumente der verschiedenen Bereiche auf konsistente "Systemgrenzen“" beziehen. Für den Bereich Umwelt existiert mit der „(Produkt-)Ökobilanz" (Life Cycle Assessment - LCA) bereits ein solches international standardisiertes Werkzeug, dessen logisches Ebenbild für den Bereich Ökonomie die „Lebenszykluskostenrechnung" (Life Cycle Costing - LCC) ist. Die „produktbezogene Sozialbilanz" (Social Life Cycle Assessment - SLCA) ist auf Grund großer methodischer Schwierigkeiten noch nicht so weit entwickelt.
\end{abstract}

\section{Einleitung}

In der politischen Diskussion über globale Entwicklung und Umwelt wird der Begriff ,Nachhaltigkeit" oftmals gebraucht und manchmal auch missbraucht. Hier soll nun ein Vorschlag zur quantitativen Nachhaltigkeitsbewertung von Produkten gemacht werden. Unter Produkten werden hier auch Dienstleistungen und Systeme verstanden. Entsprechend wird analog zur Ökobilanz ein Instrument zur Nachhaltigkeitsbewertung von Produkten vorgestellt.

Die Beschränkung der Ökobilanz auf die Umweltauswirkungen von Produkten resultierte aus der Diskussion anlässlich des ersten
SETAC-Europe-LCA-Symposiums 1991 im niederländischen Leiden. ${ }^{1}$ Natürlich war von Anfang an klar, dass eine vollständige Nachhaltigkeitsbewertung mindestens zwei weitere Dimensionen erfordert, nämlich die ökonomische und die soziale. Zehn Jahre und zwei UNWeltkonferenzen (Rio de Janeiro und Johannesburg) später rückten diese Anforderungen wieder in den Vordergrund und weckten den Wunsch nach einer Erweiterung der Ökobilanz im klassischen Sinne.

Was aber bedeutet Nachhaltigkeit eigentlich? Der Begriff wurde im Deutschen zuerst in der Forstwirtschaft verwendet. Ein Pionier auf diesem Gebiet war Hans-Carl von Carlowitz mit seinem Buch „Sylvicultura Oeconomica“, das 1713 in Leipzig veröffentlicht wurde (Carlowitz 2000). Carlowitz war zwar kein Forstwirt, aber in seiner Eigenschaft als Betriebsleiter der sächsischen Silberminen benötigte er große Mengen an Holz und stellte fest, dass sich die deutschen Wälder in keinem guten Zustand befanden. Die Forstwirtschaft blieb zeitlebens sein Hobby und von ihm stammt der Grundsatz der Forstwirtschaft, dass man auf lange Sicht nur soviel Holz einschlagen darf wie nachwächst. Er erkannte auch schon den Zusammenhang zwischen Umweltfaktoren sowie ökonomischen und sozialen Belangen. Auch wenn das Buch wegen der barocken Sprache und des Satzes in gotischen Lettern nicht gerade leichte Lektüre darstellt, ist doch diese Botschaft klar und durchaus relevant für die heutige Nachhaltigkeitsdiskussion.

Heute kennen wir den Begriff Nachhaltigkeit (oder englisch ,sustainability“) im Kontext globaler Entwicklung, wie er in der oft zitierten Definition des Brundtland-Berichtes erscheint: „Sustainable development is development that meets the needs of present without compromising the ability of future generations to meet their own needs." (WCED 1987)

Hierin wird die Verantwortung für künftige Generationen betont. Dieses hochgesteckte Ziel wird in der politischen Diskussion gerne aufgegriffen. 1992 erklärten die Vereinten Nationen in Rio de Janeiro Nachhaltigkeit zum Leitgedanken für das 21. Jahrhundert, was zehn Jahre später in Johannesburg bestätigt wurde. Dort wurde auch bereits der Bezug zum gesamten Lebenszyklus, also das ,life cycle thinking", als wichtiger Grundsatz erkannt. Jenseits 
aller politischen Absichtserklärungen bleibt jedoch die Notwendigkeit einer Quantifizierung und Operationalisierung von Nachhaltigkeit, wenn ein Missbrauch beispielsweise bei Produktvergleichen vermieden werden soll.

\section{Das Drei-Säulen-Modell}

Das auch von Seiten der Industrie gut angenommene Standardmodell ist die Drei-SäulenInterpretation von Nachhaltigkeit, die auf Englisch auch „triple bottom line“ genannt wird. Dieses sagt im Wesentlichen aus, dass zum Erreichen und natürlich auch zum Bewerten von Nachhaltigkeit ökologische, ökonomische sowie soziale Aspekte bestimmt und gegeneinander abgewogen werden müssen. Wie bereits erwähnt wurde diese Interpretation beim ersten SETAC-Europe-LCA-Symposium 1991 diskutiert, aber sie war schon früher Gegenstand der „Produktlinienanalyse“, die vom Öko-Institut 1987 in einem Buch vorgestellt wurde (Projektgruppe ökologische Wirtschaft 1987). Dies geschah im gleichen Jahr wie die Veröffentlichung des Brundtland-Berichts (WCED 1987). Die Produktlinienanalyse, deren Nachfolger kürzlich mit PROSA (,Product Sustainability Assessment" oder Produkt-Nachhaltigkeitsanalyse) vorgestellt wurde (Grießhammer et al. 2007), war damals einer der Vorläuferinnen der Ökobilanz, wobei die Wirkungsabschätzung drei Dimensionen umfasste und nicht nur eine.

Ausgehend von der weiten Akzeptanz, die auch von der ,SETAC / UNEP Life Cycle Initiative" dokumentiert wird (Remmen et al. 2007), gelangt man schnell zu folgendem Entwurf für die lebenszyklusbasierte Nachhaltigkeitsbewertung von Produkten (in Englisch: Life Cycle Sustainability Assessment $<$ LCSA $>$ ):

$$
\text { LCSA }=\mathrm{LCA}+\mathrm{LCC}+\text { SLCA }
$$

Dieses Schema wurde vor einigen Jahren als SustAss mit den drei Bestandteilen LCA, LCC und SLCA vorgestellt (Klöpffer 2003). Dabei ist LCA die Ökobilanz bzw. das Life Cycle Assessment im Sinne von SETAC und ISO, LCC das LCA-analoge Life Cycle Costing (Lebenszykluskostenrechnung) und SLCA bedeutet social LCA, d. h. die produktbezogene Sozialbilanz.
Natürlich müssen bei der Anwendung dieser Formel gewisse Voraussetzungen erfüllt sein. Als erste und wichtigste ist hier die Verwendung konsistenter, im Idealfall identischer Systemgrenzen für alle drei Bilanzen bzw. Bewertungen zu nennen. Der hier betrachtete und relevante (physikalische) Lebenszyklus eines Produktes (,from cradle to grave“) unterscheidet sich grundlegend von dem im Marketing üblichen Produktlebenszyklus, der sich über die Zeiträume der Produktentwicklung und der Produktvermarktung erstreckt. Im Idealfall ergäbe sich eine Sachbilanz als Grundlage für alle drei Dimensionen. Allerdings muss man davon ausgehen, dass das Inventar der Sozialbilanz eine stärkere regionale Auflösung erfordert als dies bei LCA und LCC nötig ist.

Der Grund, warum die produktbezogene Nachhaltigkeitsbewertung lebenszyklusbasiert sein muss, liegt auf der Hand und ist derselbe wie schon bei der Entwicklung des LCA, d. h. dass nur bei Betrachtung des gesamten Lebensweges Problemverlagerungen erkannt und vermieden werden können. Vor dem Hintergrund der Forderungen des Brundtland-Berichtes (WCED 1987) nach generationenübergreifender Fairness ist natürlich auch das Vermeiden von Problemverlagerungen in die Zukunft eine Voraussetzung für Nachhaltigkeit.

\section{Entwicklungstand der Komponenten}

\section{1 Ökobilanz - LCA}

Die Ökobilanz ist die einzige international standardisierte Umweltbewertungsmethode für Produkte. Zwei Merkmale prägen den Charakter der Ökobilanz: die analytische Betrachtung des gesamten Lebensweges ,,von der Wiege bis zur Bahre" und der Bezug zu einer funktionellen Einheit, die den Nutzen des Produktes oder der Dienstleistung quantitativ abbildet. Inzwischen wurde die ursprüngliche Serie der internationalen Standards DIN EN ISO 14040 bis 14043 (1997 bis 2000) von den neuen leicht veränderten Standards DIN EN ISO 14040 und 14044 (2006) abgelöst. Die allseits bekannte Struktur mit Festlegung des Ziels und Untersuchungsrahmens, Sachbilanz, Wirkungsabschätzung und Auswertung wurde von der SETAC gemeinsam mit der Internationalen Organisation für Normung (ISO) während der Harmonisierung und 
Standardisierung entwickelt. Die Ökobilanz ist weiterhin ein aktives Forschungsgebiet, das auch in Zukunft methodische Weiterentwicklungen erwarten lässt. Hierzu gehören beispielsweise die Definition schwieriger Wirkungskategorien, die Input/Output-Analyse und die Hybrid-Analyse, das ,consequentional“ LCA und die korrekte Anwendungen des LCA im Life Cycle Management (s. dazu auch den Beitrag von Bauer, Poganietz in diesem Heft).

\subsection{Lebenszykluskostenrechnung - LCC}

Obwohl die Lebenszykluskostenrechnung eigentlich älteren Ursprungs ist als die Ökobilanz, wurde sie bisher - außer für einige Spezialfälle - nicht standardisiert. Zurzeit arbeitet eine SETAC-Arbeitsgruppe an diesem Thema; eine dort verfasste Veröffentlichung wird demnächst erscheinen (Hunkeler, Lichtenvort und Rebitzer 2007). Die vorgeschlagene LCC-Methode lehnt sich an das LCA gemäß ISO 14040 an und umfasst den gesamten Lebenszyklus eines Produktes einschließlich Gebrauchs- und Nachnutzungsphasen. Eine Monetarisierung möglicher externer Kosten durch Umweltschäden, die in der Zukunft entstehen könnten, findet nicht statt, um Doppelzählungen zu vermeiden. Den Umweltschäden wird ja in der Wirkungsabschätzung des LCA Rechnung getragen.

In der Lebenszykluskostenrechnung gibt es keine eigenständige Wirkungsabschätzung. Das aggregierte Ergebnis sind die berechneten Kosten, bezogen auf die gewählte funktionelle Einheit in einer bestimmten geläufigen Währung. Wie auch in der Ökobilanz sollen durch die Aggregation keine Informationen verloren gehen und die genauen Analysen der Lebenszyklusphasen dokumentiert werden. Die ökobilanz-analoge Lebenszykluskostenrechnung unterscheidet sich von der üblichen ökonomischen Kostenrechnung auch dadurch, dass alle enthaltenen Kosten dem untersuchten Produktsystem zugeordnet werden und es keinen so genannten Overhead gibt. Sie ist ebenfalls von der Umweltkostenrechnung zu unterscheiden (Rikhardsson et al. 2005, Schaltegger 2007).

Die Lebenszykluskostenrechnung stellt eine sinnvolle Ergänzung zur Ökobilanz (und zur produktbezogenen Sozialbilanz) dar, denn nachhaltige Produkte sollen auch profitabel und erschwinglich sein, wenn sie auf dem Markt Akzeptanz finden sollen. Konsumentenentscheidungen basieren oftmals ausschließlich auf dem Preis eines Produktes; daher können Informationen, wie sie die Lebenszykluskostenrechnung liefert, $\mathrm{zu}$ fundierteren Kaufentscheidungen führen, weil sie z. B. auch die Nutzungsphase einschließt.

\subsection{Produktbezogene Sozialbilanz - SLCA}

Auch wenn die Idee nicht neu ist (Projektgruppe ökologische Wirtschaft 1987, O'Brian et al. 1996), so steht die produktbezogene Sozialbilanz noch am Anfang ihrer Entwicklung. Die Thematik präsentiert sich momentan als sehr lebendiges Forschungsgebiet, was sich in zahlreichen Veröffentlichungen manifestiert, von denen die aktuellsten hier kurz skizziert werden sollen.

Dreyer und Co-Autoren zielen auf die Verantwortlichkeit der involvierten Firmen ab, auch wenn die Produkte der Bezugspunkt sind (Dreyer et al. 2006). Dadurch liegt der Schwerpunkt zwangsläufig auf den Prozessen im „Vordergrund" und den daran beteiligten Personen. Die Verantwortung einer Firmenleitung für die sozialen Bedingungen in einem Betrieb steht außer Frage und dies kann im Unterschied zum umweltbezogenen LCA wichtiger sein als die eingesetzten Prozesse. Zur Modellierung der Wirkungsabschätzung in der SLCA werden die „Menschenwürde und das Wohlergehen” (Human Dignity and Well-being) als neues Schutzgut vorgeschlagen (Dreyer et al. 2006, S. 93). Dieses soll die existierenden Schutzgüter des LCA ergänzen, allerdings werden Überschneidungen mit dem Schutzgut ,menschliche Gesundheit" eingeräumt. Normative Basis sollen universelle Normen (wie die Erklärung der Menschenrechte) sowie lokale und länderspezifische Normen sein, die die sozioökonomischen Verhältnisse und Ziele repräsentieren. Zur Modellierung auf „Midpoint”-Ebene werden die ILO-Konventionen (International Labour Organisation) herangezogen.

Weidema schließt Elemente der KostenNutzen-Analyse (Cost Benefit Analysis - CBA) mit ein und schlägt "Quality Adjusted Life Years“ (QALY) als Maß für menschliche Gesundheit und menschliches Wohlbefinden vor. 
(Weidema 2006). Auch Norris beschäftigt sich mit sozialen und sozioökonomischen Wirkungen, die zu gesundheitlichen Beeinträchtigungen führen (Norris 2006). Als Internet-basiertes Instrument soll Life Cycle Attribute Assessment (LCAA) hier die klassischen lebenszyklusbasierten Bewertungsmethoden ergänzen. Labuschagne und Brent streben die Vollständigkeit des Indikatorensatzes an. Ihre Methode scheint jedoch nicht in erster Linie auf die Produktbewertung abzuzielen (Labuschagne, Brent 2006).

Hunkeler löst das Problem des Bezuges der sozialen Wirkungen auf die funktionelle Einheit, indem er die anteilige Arbeitszeit zur Produktion des Zielproduktes zur Quantifizierung benutzt (Hunkeler 2006). Die Arbeitszeit kann Bestandteil der Sachbilanz sein, wobei aber eine regionale Differenzierung erfolgen sollte. Diese Regionalisierung ist hier von größerer Bedeutung als in der Ökobilanz üblich. Mit seiner Methode will Hunkeler zeigen, dass SLCAs auf einer präzisen Methodik basieren, mit Sachbilanzdaten verknüpft, mit Hilfe von Charakterisierungsfaktoren berechnet sowie mit Datenbanken verknüpft werden können, die die geographische Verteilung der Auswirkungen eines Produktes abschätzen. Für jeden Einzelprozess werden in der Sachbilanz die Arbeitsstunden, bezogen auf die jeweils relevanten geographischen Regionen, ermittelt. Aus regionalspezifischen Daten werden ,midpoint”-Charakterisierungsfaktoren berechnet, die angeben, mit wie viel Arbeitsstunden welcher Wohlstand finanziert werden kann. Als mögliche Bereiche werden hier „Wohnen“, „Zugang zum Gesundheitswesen“, „Bildung" und „Grundbedürfnisse“ genannt.

Verschiedene andere in jüngster Zeit entwickelte SLCA-Methoden gründen in der Ökoeffizienzanalyse, einer Kombination von vereinfachten Ökobilanzen und Lebenszykluskostenrechnungen. Saling und Co-Autoren fügen eine soziale Komponente zur Ökoeffizienzanalyse der BASF, was zur SEEbalance ${ }^{\circledR}$ führt $^{2}$ (Saling, Gensch et al. 2007). Dadurch wird aus den zweidimensionalen Ökoeffizienzdiagrammen ein Kubus, der die Position des Produktes in Bezug auf alle drei Dimensionen der Nachhaltigkeit anzeigt. Da die Methode von wertebezogenen Gewichtungsfaktoren abhängt, sollte sie nach ISO 14044 nur für interne Zwecke verwendet werden.
„Life Cycle Working Time“ (LCTW) steht für die Einbeziehung arbeitsplatzbezogener sozioökonomischer Aspekte in die LCASoftware „GaBi“, die damit neben LCA und LCC auch die dritte Komponente der Nachhaltigkeit lebenszyklusbezogen berücksichtigt. Von Grießhammer und Co-Autoren stammt eine Machbarkeitsstudie für die SETAC/ UNEP-Initiative zur Integration sozialer Aspekte in die Ökobilanz (Grießhammer et al. 2006). Weil sich viele soziale Indikatoren nicht quantifizieren lassen, benutzt man ein qualitatives Bewertungsschema in Ergänzung zu quantitativen Ergebnissen. Pesonen stellte kürzlich Sustainability SWOTs ${ }^{3}$ als vereinfachte Form der SLCA vor (Pesonen 2007). Schließlich gibt es von Jørgensen und CoAutoren eine Übersicht zu einschlägigen Veröffentlichungen und "Grauer Literatur" zur SLCA (Jørgensen et al. 2007).

Es ist sicherlich zu früh für eine Standardisierung der produktbezogenen Sozialbilanz, jedoch könnte ein gewisses Maß an Harmonisierung erreicht werden, wenn die verschiedenen Ansätze in Fallstudien verglichen würden. Wie auch im Finanzwesen könnte es sich als nützlich erweisen, unterschiedliche Indikatoren zur Beurteilung der verschiedenen Aspekte der SLCA zu haben. So könnten Erfahrungen gesammelt werden und die passende(n) Methode(n) würden sich abzeichnen. Bezüglich der Wirkungen und ihrer Indikatoren sollte man nicht vergessen, dass es aus gutem Grund auch in der Wirkungsabschätzung der Ökobilanz keine absolut gültige Liste gibt.

Die Hauptschwierigkeiten in der produktbezogenen Sozialbilanz lassen sich über folgende Fragen charakterisieren:

1. Wie lassen sich bestehende Indikatoren mit der funktionellen Einheit des untersuchten Systems verknüpfen?

2. Wie erhält man die spezifischen Daten zur (notwendigen) regionalen Auflösung der SLCA?

3. Wie entscheidet man zwischen vielen qualitativen Indikatoren und einigen wenigen quantifizierbaren (z. B. über die Inventarisierung von Arbeitszeit pro funktionelle Einheit)?

4. Wie quantifiziert man die Wirkungen korrekt? 
Der letzte Punkt ist wohl der schwierigste, und in der Tat ist auch in der Ökobilanz die Quantifizierung aller Wirkungen nicht möglich. So sei beispielsweise daran erinnert, dass es für die wichtige Wirkungskatgorie Biodiversität auch noch keinen passenden allgemein akzeptierten Indikator gibt.

\section{Ein Life Cycle Assessment oder drei?}

Es gibt mindestens zwei Optionen, wie sich die Lebenszykluskostenrechnung und die produktbezogene Sozialbilanz in die Nachhaltigkeitsbewertung von Produkten integrieren lassen:

\section{Option 1: LCSA = LCA + LCC + SLCA}

Option 1 basiert auf drei getrennten lebenszyklusbasierten Bewertungen mit konsistenten, idealerweise identischen Systemgrenzen - wie bereits in der Einleitung vorgeschlagen. Diese beiden noch nicht genormten Methoden sollten zukünftig standardisiert oder zumindest harmonisiert und an die Ökobilanz (LCA) angepasst sein.

Eine Gewichtung zwischen den drei „Säulen" oder Dimensionen sollte nicht stattfinden. So bleibt Transparenz gewahrt, was sicher als großer Vorteil dieser Option anzusehen ist. Die Zuordnung von Vor- und Nachteilen in vergleichenden Bewertungen ist klar; es gibt - und sollte es auch nicht - keinen Ausgleich zwischen den drei Dimensionen Ökologie, Ökonomie und Soziales.

Option 2: LCSA = „LCAneu“ (einschließlich LCC und SLCA als zusätzliche Wirkungskategorien in der Wirkungsabschätzung)

Option 2 bedeutet, dass ausgehend von einer Sachbilanz bis zu drei (LCC hat keine formale Wirkungsabschätzung) Wirkungsabschätzungen erstellt werden, die sich vermutlich auf einen Satz von Schutzgütern beziehen. Der Vorteil ist hier, dass nur ein Sachbilanzmodell in der Festlegung des Ziels und Untersuchungsrahmens definiert werden muss. Andererseits kann auch das Inventar einer Ökobilanz für die produktbezogene Sozialbilanz verwendet werden, wie in der Methode von Hunkeler vorgestellt (Hunkeler 2006).

Für beide Optionen gibt es Fürsprecher, wobei die mögliche künftige Erweiterung der ISO 14040er Serie die Diskussion entscheidend mitbestimmt. Daher stellt sich folgende wichtige Frage: Ist Option 2 kompatibel zu ISO 14040? Dieser Standard sagt hierzu: „Eine Ökobilanz bezieht sich auf die Umweltaspekte und -wirkungen eines Produktsystems. Üblicherweise liegen ökonomische und soziale Aspekte und Wirkungen außerhalb des Untersuchungsrahmens der Ökobilanz" (ISO 14040, Abschnitt 4.1.3). Bereits in der Einleitung heißt es wörtlich: „Üblicherweise werden ökonomische oder soziale Aspekte eines Produktes bei Ökobilanzen nicht berücksichtigt, der Ökobilanz-Ansatz und die in dieser internationalen Norm beschriebene Methodik können jedoch auch auf diese anderen Aspekte angewendet werden." (dies., Einleitung)

Diese Zitate aus DIN EN ISO 14040 sprechen eindeutig für Option 1 und eine separate Standardisierung der LCA-analogen LCC und SLCA wäre die logische Konsequenz. Andererseits könnten die Standards ISO 14040 und 14044 in Zukunft wieder geändert werden, um Option 2 ISO-konform zu ermöglichen. Dies würde allerdings auch zur Folge haben, dass der ohnehin schon lange Standard ISO 14044 noch um detaillierte "Sollte“- und „Muss“Bestimmungen für LCC und SLCA erweitert werden müsste.

\section{Schlussfolgerungen}

Wenn davon auszugehen ist, dass die Menschheit nur dann eine Überlebenschance hat, wenn ein nachhaltiges Entwicklungsmodell etabliert wird, muss Nachhaltigkeit auf allen Ebenen verwirklicht werden. Dies umfasst regionale, nationale und globale Dimensionen (nicht zu vergessen die kommunalen Ebenen). Der hier formulierte Vorschlag betrifft den verhältnismäßig bescheidenen Aspekt der Produkte und Prozesse. Life Cycle Management wird primär von Industrieseite umgesetzt und kann von gut informierten Verbrauchern beeinflusst werden. So kann man Schritt für Schritt auch ohne direkten Einfluss der Politik vorankommen, allerdings nicht ohne das Zutun der Wirtschaft. Dies ist ein starkes Argument für die Aufnahme der Lebenszykluskostenrechnung. Ohne soziale Gerechtigkeit kann langfristig keine Entwicklung bestehen, deshalb muss auch die 
produktbezogene Sozialbilanz weiterentwickelt und berücksichtigt werden.

Es wird oft gesagt, dass schon das Denken in Lebenszyklen ausreicht und dass die Bewertungsmethoden einfach und nicht immer quantitativ sein müssten. Dies mag beim Aufzeigen von Brennpunkten stimmen, aber sicher nicht bei der fundierten Entscheidungsfindung weiterhelfen: Wenn verschiedene Lösungsvorschläge gemacht werden, braucht man quantitative Methoden. Es ist die Stärke des LCA, dass Quantifizierung möglich ist und dieser Vorteil sollte auch bei der Ergänzung ökonomischer und sozialer Aspekte erhalten bleiben. Bei der Lebenszykluskostenrechnung wird dies relativ einfach sein, aber schwierig für die produktbezogene Sozialbilanz. Im Hinblick auf die große Bedeutung des Zieles sollten gleich große Anstrengungen unternommen werden, um die nötigen Bewertungswerkzeuge zur Verfügung stellen und kontinuierlich verbessern zu können.

\section{Anmerkungen}

1) Die SETAC (Society of Environmental Toxicology and Chemistry) ist eine weltweit agierende wissenschaftliche Gesellschaft (s. http://www. setac-glb.org/About.htm) (Anm. d. Red.).

2) Bei der Ökoeffizienzmethode der BASF handelt es sich im Umweltteil um eine vereinfachte Ökobilanz, in der ökonomischen Bewertung um Lebenszykluskosten, die oft aus den Preisen abgeleitet werden (Saling, Kicherer et al. 2002). Die Vereinfachungen erlauben die rasche Erstellung von Ökoeffizienzanalysen und damit auch die Analyse vieler Produkte. Den beiden genannten Analysen wurde nun im Sinne der Dreisäulen-Theorie eine soziale Komponente (SEEbalance) hinzugefügt.

3) Die SWOT-Analyse (engl. Akronym für Strengths $<$ Stärken>, Weaknesses <Schwächen>, Opportunities $<$ Chancen $>$ und Threats $<$ Gefahren $>$ ) ist ein Werkzeug des strategischen Managements, wird aber auch für formative Evaluationen und Qualitätsentwicklung von Programmen (z. B. im Bildungsbereich) eingesetzt (http://de.wikipedia.org).

\section{Literatur}

Carlowitz, H.C., 2000: Sylvicultura Oeconomica Naturmäßige Anweisung zur Wilden Baum-Zucht. Reprint der Ausgabe Leipzig 1713, Freiberg
Dreyer, L.C.; Hauschild, M.; Schierbeck, J., 2006: A Framework for Life Cycle Impact Assessment. In: International Journal of Life Cycle Assessment 11/2 (2006), S. 88-97

Grießhammer, R.; Benoît, C.; Dreyer, L.C. et al., 2006: Feasibility Study 2006: Integration of Social Aspects into LCA. Discussion paper from UNEPSETAC Task Force Integration of Social Aspects in LCA. Freiburg

Grießhammer, R.; Buchert, M.; Gensch, C.-O. et al., 2007: PROSA - Product Sustainability Assessment. Leitfaden. Freiburg: Öko-Institut e.V.; http://www.prosa.org

Hunkeler, D., 2006: Societal LCA Methodology and Case Study. In: International Journal of Life Cycle Assessment 11 (2006), S. 371-382

Hunkeler, D.; Lichtenvort, K.; Rebitzer, G. (eds.), 2007: Environmental Life Cycle Costing. SETAC. Im Erscheinen

Hunkeler, D.; Rebitzer, G., 2005: The Future of Life Cycle Assessment. In: International Journal of Life Cycle Assessment 10/5 (2005), pp. 305-308

ISO 14040, 2006: Environmental management Life cycle assessment - Principles and framework

Jørgensen, A.; Le Bocq, A.; Nazarkina, L. et al., 2007: Methodologies for Social Life Cycle Assessment - A Review. Accepted for publication in International Journal of Life Cycle Assessment

Klöpffer, W., 2003: Life-Cycle Based Methods for Sustainable Product Development. Editorial for the Life Cycle Management, Section in International Journal of Life Cycle Assessment 8 (2003), S. $157-159$

Labuschagne, C.; Brent, A.C., 2006: Social Indicators for Sustainable Project and Technology Life Cycle Management in the Process Industry. In: International Journal of Life Cycle Assessment 11 (2006), S. 3-15

Norris, G.A., 2006: Social Impacts in Product Life Cycles. Towards Life Cycle Attribute Assessment. In: International Journal of Life Cycle Assessment 11 (Sp. Iss. 1, 2006), S. 97-104

O'Brian, M.; Doig, A.; Clift, R., 1996: Social and Environmental Life Cycle Assessment (SELCA). In: International Journal of Life Cycle Assessment 1 (1996), S. 231-237

Pesonen, H.-L., 2007: Sustainability SWOTs - New Method for Summarizing Product Sustainability Information for Business Decision Making. Platform presentation at the $3^{\text {rd }}$ International Conference on Life Cycle Management Zurich, 27-29 August 2007

Projektgruppe ökologische Wirtschaft (Hg.), 1987: Produktlinienanalyse: Bedürfnisse, Produkte und ihre Folgen. Köln 
Remmen, A.; Jensen, A.A.; Frydendal, J., 2007: The Triple Bottom Line - the Business Case of Sustainability. In: Life Cycle Management. A Business Guide to Sustainability. UNEP / SETAC Life Cycle Initiative, S. 10-11

Rikhardsson, P.; Bennett, M.; Bouma, J. et al. (eds.), 2005: Implementing Environmental Management Accounting: Status and Challenges. Berlin

Saling, P.; Gensch, C.-O.; Kreisel, G. et al., 2007: Entwicklung der Nachhaltigkeitsbewertung SEEbalance ${ }^{\circledR}$ - im BMBF-Projekt „Nachhaltige Aromatenchemie". Karlsruhe (Karlsruher Schriften zur Geographie und Geoökologie)

Saling, P.; Kicherer, A.; Dittrich-Krämer, B. et al., 2002: Eco-efficiency Analysis by BASF: The method. In: International Journal of Life Cycle Assessment 7 (2002), S. 203-218

Schaltegger, S. (ed.), 2007: Environmental Management Accounting for Cleaner Production. Im Erscheinen

WCED - World Commission on Environment and Development, 1987: Our Common Future. Oxford

Weidema, B.P., 2006: The Integration of Economic and Social Aspects in Life Cycle Impact Assessment. In: International Journal of Life Cycle Assessment 11 (Sp. Iss. 1, 2006), S. 89-96

\section{Kontakt}

Prof. Dr. Walter Klöpffer

LCA Consult \& Review

Am Dachsberg 56 E, 60435 Frankfurt a. M.

E-Mail: walter.kloepffer@t-online.de
Realistic Eco-Efficiency Analysis

Why We Need Better

Eco-Efficiency Analysis

From Technological Optimism

to Realism

\author{
by Gjalt Huppes, CML
}

Eco-efficiency analysis relates two pillars of sustainability, the economic and the environmental one. There are several options for specifying eco-efficiency, as a partial or more encompassing concept. When using technology specification as the basis for eco-efficiency analysis, there is an inbuilt tendency towards unjustified optimism, as other societal mechanisms detract from the technology potential. A more systematic approach to modelling is required to arrive at a more realistic analysis, both lining the micro level analysis to the macro level sustainability consequences for society and reckoning with the relevant mechanism in society of economic, cultural, institutional and political nature. With more realistic modelling, more realistic requirements on the trade off between economy and environment at a micro level can be formulated. A substantial research programme is required for this purpose, with substantial efforts at standardisation. Work in the EU project CALCAS is addressing such research framing questions.

\section{Introduction}

The race between economic growth and environmental improvement is an open one. In some respects, the environment improves faster, as in terms of reduced toxic emissions. In other respects, economic growth is larger than environmental improvements per average unit of consumption, and hence the environment deteriorates. Main examples are global warming and land use shifts eating up nature areas. Together these constitute main factors for the fast reduction in biodiversity. Due to global interconnectedness of production processes, and as low hanging fruits have already been picked, measures for improving one environmental aspect impinge on other environmental aspects. Also, a fundamental sub-optimality is created by the 